\title{
Scalar perturbations in Randall-Sundrum braneworld cosmology
}

\author{
Antonio CARDOSO* \\ Institute of Cosmology \& Gravitation, University of Portsmouth, Portsmouth PO1 2EG, UK \\ E-mail: Antonio.Cardoso@port.ac.uk
}

\begin{abstract}
We study the evolution of scalar perturbations in the radiation-dominated era of Randall-Sundrum braneworld cosmology by numerically solving the coupled bulk and brane master wave equations. We find that density perturbations with wavelengths less than a critical value (set by the bulk curvature length) are amplified during horizon re-entry. Conversely, we explicitly confirm from simulations that the spectrum is identical to GR on large scales. Although this magnification is not relevant for the cosmic microwave background or measurements of large scale structure, it may have some bearing on the formation of primordial black holes in Randall-Sundrum models.
\end{abstract}

Cargèse Summer School: Cosmology and Particle Physics Beyond the Standard Models

July 30 - August 11, 2007

Institut d'Etudes Scientifiques de Cargese

\footnotetext{
*Speaker.

${ }^{\dagger}$ Based on the work done in collaboration with Takashi Hiramatsu, Kazuya Koyama and Sanjeev S. Seahra, reported in [1].
} 


\section{Introduction}

The Randall-Sundrum (RS) braneworld model [2] postulates that our observable universe is a thin 4-dimensional hypersurface residing in 5-dimensional anti-de Sitter (AdS) space. Ordinary matter degrees of freedom are assumed to be confined to the brane, while gravitational degrees of freedom are allowed to propagate in the full 5-dimensional bulk. The warping of AdS space allows us to recover ordinary general relativity (GR) at distances greater than the curvature radius of the bulk $\ell$. Current laboratory tests of Newton's law constrain $\ell$ to be less than around $0.1 \mathrm{~mm}$ [3].

It is well known that the Friedmann equation governing the expansion of the brane universe differs from general relativity by a correction of order $\rho / \sigma$, where $\rho$ is the energy density of the brane matter and $\sigma \gtrsim(\mathrm{TeV})^{4}$ is the brane tension. The magnitude of this correction defines the "high-energy" regime of braneworld cosmology as the era when $\rho \gtrsim \sigma$ or equivalently $H \ell \gtrsim 1$, where $H$ is the Hubble parameter.

The equations of motion governing fluctuations of the model are found to differ from GR in two principal ways at early times: First, they acquire $\mathscr{O}(\rho / \sigma)$ high-energy corrections similar to those found in the Friedmann equation. By themselves, such corrections are not difficult to deal with: they just modify the second-order ordinary differential equations (ODEs) governing perturbations in GR. But the second type of modification is more problematic: perturbations on the brane are also coupled to fluctuations of the 5-dimensional bulk geometry, which are collectively known as the "Kaluza Klein" (KK) degrees of freedom of the model. The KK modes are governed by master partial differential equations (PDEs) defined throughout the AdS bulk [4, 5]. The only known way of solving this system of equations on all scales simultaneously is by direct numerical solution.

The purpose of the paper is to numerically solve for the behaviour of scalar perturbations in the radiation-dominated regime of braneworld cosmology. We use two different numerical codes recently developed in Refs. [6, 7], which gives us the ability to confirm the consistency of our numeric results via two independent algorithms. We are ultimately interested in finding the matter transfer function in the radiation era, and also determining the relative influence of KK and highenergy effects on the density perturbations. Heuristically, we may expect the KK modes to amplify high-energy/small-scale density perturbations. The reason is that we know that the gravitational force of attraction in the RS model is stronger than in GR on scales less than $\ell[2,8]$. This implies that modes with a physical wavelength smaller than $\ell$ during horizon crossing will be amplified due to the KK enhancement of the gravitational force. However, this physical reasoning needs to be tested with numeric simulations.

\section{Scalar perturbations}

It has been shown in Refs. $[4,5]$ that scalar-type perturbations of the bulk geometry are governed by a single gauge invariant master variable $\Omega$. This bulk master variable satisfies the following wave equation

$$
0=-\frac{\partial^{2} \Omega}{\partial \tau^{2}}+\frac{\partial^{2} \Omega}{\partial z^{2}}+\frac{3}{z} \frac{\partial \Omega}{\partial z}+\left(\frac{1}{z^{2}}-k^{2}\right) \Omega
$$


and a boundary condition on the brane

$$
\left[\partial_{n} \Omega+\frac{1}{\ell}\left(1+\frac{\rho}{\sigma}\right) \Omega+\frac{6 \rho a^{3}}{\sigma k^{2}} \Delta\right]_{\mathrm{b}}=0 .
$$

The density contrast on the brane $\Delta$ satisfies the wave equation

$$
\begin{gathered}
\frac{d^{2} \Delta}{d \eta^{2}}+\left(1+3 c_{s}^{2}-6 w\right) H a \frac{d \Delta}{d \eta}+\left[c_{s}^{2} k^{2}+\frac{3 \rho a^{2}}{\sigma \ell^{2}} A+\frac{3 \rho^{2} a^{2}}{\sigma^{2} \ell^{2}} B\right] \Delta=-\frac{k^{2} \Gamma}{\rho}+\frac{k^{4}(1+w) \Omega_{\mathrm{b}}}{3 \ell a^{3}}, \\
A=6 c_{s}^{2}-1-8 w+3 w^{2}, \quad B=3 c_{s}^{2}-9 w-4 .
\end{gathered}
$$

Here, we have defined the sound speed $c_{s}^{2}=\delta p / \delta \rho$ and the equation of state $w=p / \rho$ (where $\rho$ and $p$ are the energy density and pressure of the brane fluid, respectively), $\Gamma$ is the entropy perturbation of the brane matter and $\eta$ is the conformal time along the brane. In this paper we assume that the matter anisotropic stress vanishes. The above ODE, the bulk wave equation (2.1) and the boundary condition (2.2) comprise a closed set of equations for $\Delta$ and $\Omega$. Note that in the low energy universe, we can neglect $\mathscr{O}\left(\rho^{2} / \sigma^{2}\right)$ terms. If we also set $\Omega_{\mathrm{b}}=0$ we obtain the standard 4-dimensional dynamical equation for $\Delta$; hence, we recover GR at low energies.

\section{Numeric analysis and discussion}

For the rest of the paper, we will restrict ourselves to the case of a radiation-dominated brane with $w=1 / 3$. We define the "*" epoch as the moment in time when a mode with wavenumber $k$ enters the Hubble horizon, $k=H_{*} a_{*}$. Another important era is the critical epoch, the transition between high and low energy regimes, when $H_{c} \ell=1$ and the radiation density has its critical value $\rho_{c} / \sigma=\sqrt{2}-1$. Generally speaking, we call modes with $k>k_{\mathrm{c}}$ "supercritical" and modes with $k<k_{\mathrm{c}}$ "subcritical". The scale defined by the critical mode in today's universe corresponds, for $\ell=0.1 \mathrm{~mm}$, to a scale of $\sim 10$ astronomical units (AU), which is incredibly tiny by cosmological standards.

In Fig. 1, we plot the predictions of GR, the 4-dimensional effective theory (where all $\mathscr{O}(\rho / \sigma)$ corrections to GR are retained, but the bulk effects are removed by artificially setting $\Omega=0$ ), and the full 5-dimensional simulations for the behaviour of the curvature perturbation on uniform density slices $\zeta$ and the density contrast $\Delta$ for a supercritical mode. Since in any given model we expect the primordial value of the curvature perturbation to be fixed by inflation, it makes physical sense to normalize the waveforms from each theory such that $\zeta_{\mathrm{SD}} \approx \zeta_{\mathrm{eff}} \approx \zeta_{\mathrm{GR}} \approx 1$ for $a \ll a_{*}$. When this is enforced we see that the effective theory predicts a larger final amplitude for the density perturbation than GR. Furthermore, the final amplitude in the 5-dimensional simulation is larger than both of the other theories. From this we can infer that, as we expected, both $\mathscr{O}(\rho / \sigma)$ and KK effects induce enhancement in the amplitude of perturbations.

As in Fig. 1, let the final amplitudes of the density perturbation with wavenumber $k$ be $\mathscr{C}_{5 \mathrm{D}}(k)$, $\mathscr{C}_{\text {eff }}(k)$ and $\mathscr{C}_{\mathrm{GR}}(k)$ for the 5-dimensional, effective and GR theories, respectively. Then, we define enhancement factors as

$$
\mathscr{Q}_{\text {eff }}(k)=\frac{\mathscr{C}_{\text {eff }}(k)}{\mathscr{C}_{\mathrm{GR}}(k)}, \quad \mathscr{Q}_{\mathscr{E}}(k)=\frac{\mathscr{C}_{5 \mathrm{D}}(k)}{\mathscr{C}_{\text {eff }}(k)}, \quad \mathscr{Q}_{5 \mathrm{D}}(k)=\frac{\mathscr{C}_{5 \mathrm{D}}(k)}{\mathscr{C}_{\mathrm{GR}}(k)} .
$$



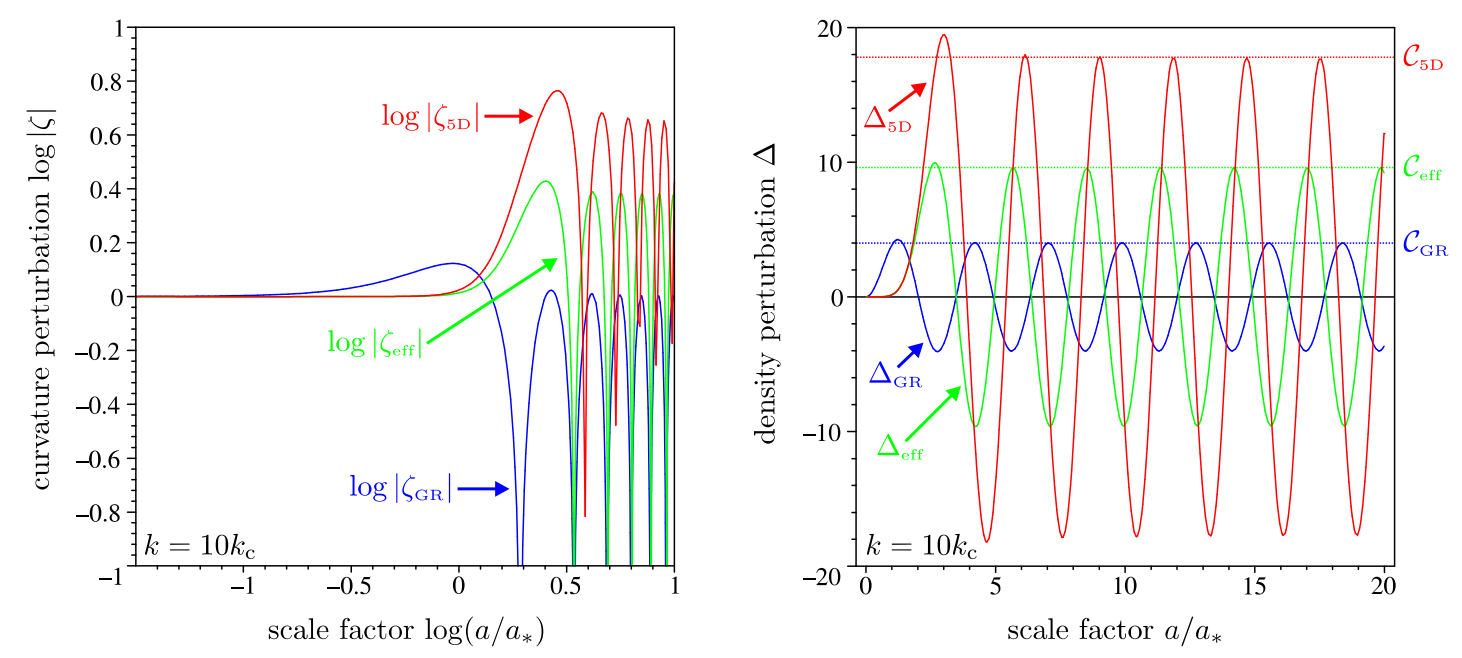

Figure 1: A comparison of the behaviour of the curvature perturbation $\zeta$ (left) and the density perturbation $\Delta$ (right) in the full 5-dimensional theory including KK contributions (5D), the effective 4-dimension theory including $\mathscr{O}(\rho / \sigma)$ corrections (eff), and ordinary general relativity (GR). The waveforms for each theory are normalized such that $\zeta=1$ on superhorizon scales.

It follows that $\mathscr{Q}_{\text {eff }}(k)$ represents the $\mathscr{O}(\rho / \sigma)$ enhancement to the density perturbation, $\mathscr{Q}_{\mathscr{E}}(k)$ gives the magnification due to KK modes, while $\mathscr{Q}_{5 \mathrm{D}}(k)$ gives the total 5-dimensional amplification over the GR case. These enhancement factors are shown in the left panel of Fig. 2. We can see that they all increase as the scale is decreased, and that they all approach unity for $k \rightarrow 0$, which means we recover general relativity on large scales. For all wavenumbers we see $\mathscr{Q}_{\text {eff }}>\mathscr{Q}_{\mathscr{E}}>1$, which implies that the amplitude magnification due to the $\mathscr{O}(\rho / \sigma)$ corrections is always larger than that due to the KK modes. Interestingly, the $\mathscr{Q}$-factors appear to approach asymptotically constant values for large $k$.

Now we consider a transfer function $T(k)$ that will tell us how the initial spectrum of curvature perturbations $\mathscr{P}_{\zeta}^{\text {inf }}$ maps onto the spectrum of density perturbations $\mathscr{P}_{\Delta}$ at some low energy epoch within the radiation era characterized by the conformal time $\eta>\eta_{\mathrm{c}}$. It is customary to normalize transfer functions such that $T(k ; \eta) \underset{0}{\stackrel{k}{\longrightarrow}} 1$, which leads us to the following definition

$$
T(k ; \eta)=\frac{9}{4}\left[\frac{k}{H(\eta) a(\eta)}\right]^{-2} \frac{\Delta_{k}(\eta)}{\zeta_{k}^{\text {inf }}} .
$$

Here, $\zeta_{k}^{\text {inf }}$ is the primordial value of the curvature perturbation and $\Delta_{k}(\eta)$ is the maximum amplitude of the density perturbation in the epoch of interest. We know that we recover the GR result in the extreme small scale limit, which gives the transfer function the correct normalization. In the righthand panel of Fig. 2, we show the transfer functions derived from GR, the effective theory and the 5-dimensional simulations. As expected, the $T(k ; \eta)$ for each formulation match one another on subcritical scales $k<k_{\mathrm{c}}$. However, on supercritical scales we have $T_{\mathrm{SD}}>T_{\text {eff }}>T_{\mathrm{GR}}$. Our results are robust against modifications of the initial data for simulations.

The amplitude enhancement of perturbations is important on comoving scales $\lesssim 10 \mathrm{AU}$, which are far too small to be relevant to present-day/cosmic microwave background measurements of the 

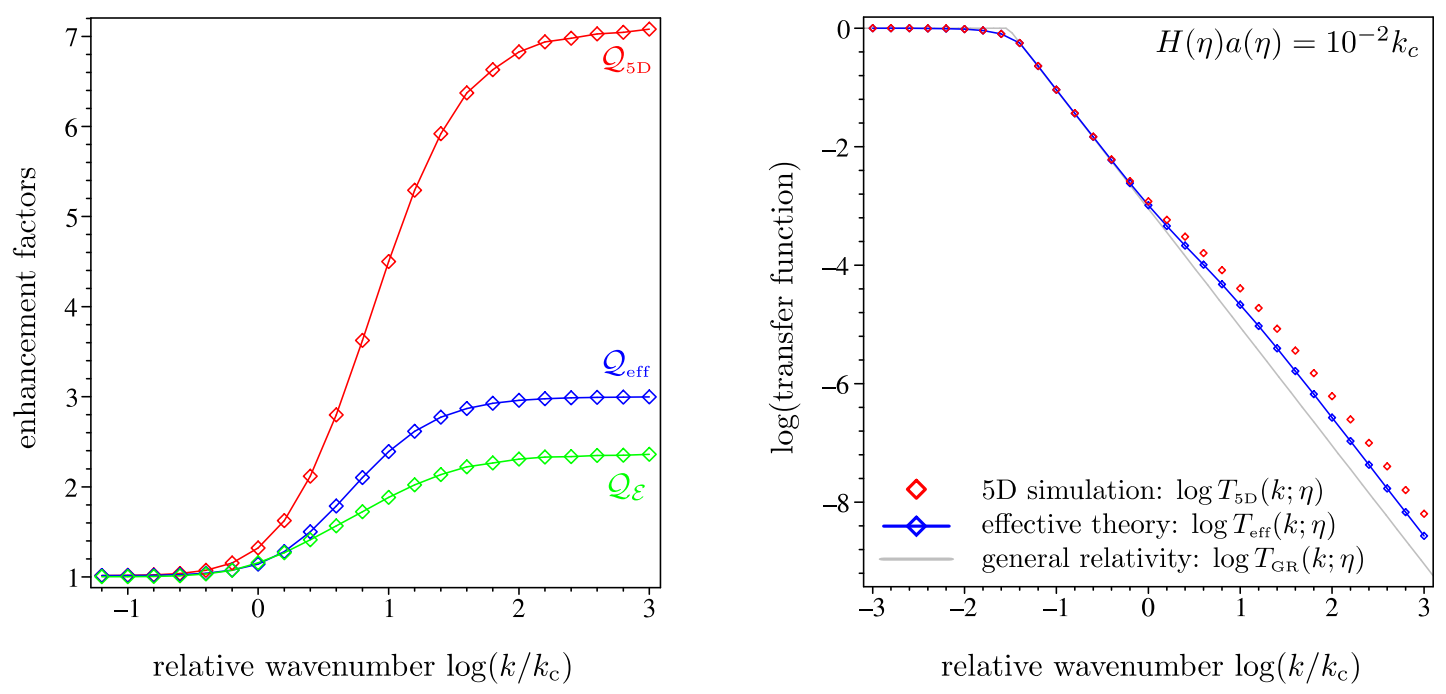

Figure 2: Density perturbation enhancement factors (left) and transfer functions (right) from simulations, effective theory, and general relativity. The transfer functions in the right panel are evaluated at a given subcritical epoch in the radiation dominated era.

matter power spectrum. However, it may have an important bearing on the formation of compact objects such as primordial black holes and boson stars at very high energies, i.e. the greater gravitational force of attraction in the early universe will create more of these objects than in GR. We know that the abundance of primordial black holes can be constrained by big bang nucleosythesis and observations of high-energy cosmic rays, so it would be interesting to see if the kind of enhancement of density perturbations predicted in this paper can be used to derive new limits on Randall-Sundrum cosmology.

\section{Acknowledgments}

I am supported by FCT (Portugal) PhD fellowship SFRH/BD/19853/2004.

\section{References}

[1] A. Cardoso, T. Hiramatsu, K. Koyama and S. S. Seahra, arXiv:0705.1685 [astro-ph].

[2] L. Randall and R. Sundrum, Phys. Rev. Lett. 83 (1999) 4690 [arXiv:hep-th/9906064].

[3] D. J. Kapner, T. S. Cook, E. G. Adelberger, J. H. Gundlach, B. R. Heckel, C. D. Hoyle and H. E. Swanson, Phys. Rev. Lett. 98 (2007) 021101 [arXiv:hep-ph/0611184].

[4] S. Mukohyama, Phys. Rev. D 62 (2000) 084015 [arXiv:hep-th/0004067].

[5] H. Kodama, A. Ishibashi and O. Seto, Phys. Rev. D 62 (2000) 064022 [arXiv:hep-th/0004160].

[6] T. Hiramatsu and K. Koyama, JCAP 0612 (2006) 009 [arXiv:hep-th/0607068].

[7] A. Cardoso, K. Koyama, A. Mennim, S. S. Seahra and D. Wands, Phys. Rev. D 75 (2007) 084002 [arXiv:hep-th/0612202].

[8] J. Garriga and T. Tanaka, Phys. Rev. Lett. 84 (2000) 2778 [arXiv:hep-th/9911055]. 